International AIH Survey on Patients' Views and Experiences collected information about the support mechanisms that are currently available.

Methods Clinicians and patient representatives designed the survey. An electronic weblink was disseminated by AIH Support, LiverNorth and the British Liver Trust, for anonymous data collection from patients in any country. Thematic qualitative and descriptive data analyses were undertaken.

Results A total of 270 responses were received (median age 55 [range 17-83 years], 94\% female). Almost half (49\%) reported being embarrassed to tell people that they have AIH. The majority attributed this to the stigma surrounding liver disease and the perception that their disease is self-inflicted or infectious (many suggested a name change from hepatitis). $53 \%$ worry about their disease either all or a lot of the time and $56 \%$ worry about the effect their AIH medication has on them either all or a lot of the time. A third of patients reported that worrying about the future is the most difficult aspect of living with AIH. Fatigue was the most frequent answer when asked about frustrations and difficulties associated with $\mathrm{AIH}$.

In terms of patient support, 47\% had accessed patient groups, with $79 \%$ being AIH-specific. This may be influenced by the route of survey dissemination and higher than in the whole patient community. The average helpfulness score was 7.9 (1 not helpful - 10 extremely helpful). Only 19\% had access to a specialist liver nurse. Key themes were better access to specialist care, improved communication, proper acknowledgement of symptoms and more research to find better treatments with fewer side effects and, ultimately, a cure.

Conclusions Medical care often focusses on disease control but this data highlights important factors that impact on patients' experiences of $\mathrm{AIH}$. The stigma associated with liver disease and not feeling adequately informed about their treatments or prognosis leads to significant anxiety. A more holistic approach to care is needed and signposting towards support groups can be very valuable for patients.

\section{P185 RADIOLOGICAL RESPONSE TO TRANS-ARTERIAL CHEMOEMBOLISATION (TACE) DETERMINES OUTCOME IN PATIENTS WITH HEPATO-CELLULAR CARCINOMA (HCC)}

${ }^{1}$ Nada Elamin*, ${ }^{1}$ Chris Keegan, ${ }^{1}$ Elizabeth Bolland, ${ }^{1}$ Cyril Sieberhagen, ${ }^{1}$ Richard Sturgess, ${ }^{1}$ Tim Cross, ${ }^{2}$ Olusola Faluyi, ${ }^{2}$ Daniel Palmer, ${ }^{1}$ Gaurav Sundar, ${ }^{1}$ Rob Davies, ${ }^{1}$ Elizabeth O'Grady, 'Nick Stern. 'Liverpool University Hospital NHS Foundation Trust, Liverpool, UK; ${ }^{2}$ The Clatterbridge Cancer Centre, Liverpool, UK

\subsection{6/gutjnl-2020-bsgcampus.260}

Introduction TACE is considered standard of care treatment for patients with Barcelona Clinic for Liver Cancer (BCLC) stage B HCC. Imaging post treatment determines further treatment offered, based on response. Radiological response is categorised as complete response (CR), partial response (PR), stable disease (SD) or progressive disease (PD). Outcome can be predicted pre-TACE or following the first treatment based on a number of parameters (HAP and ART ref). We wanted to demonstrate the survival differences based on radiological response to TACE alone when looking at patient outcome.

Methods All HCC patients treated with TACE at our regional centre since 2010 were included for analysis. Demographic details were obtained with proportion of cirrhotic patients, aetiology of liver disease, BCLC stage, Child Pugh Score and
Hepatoma Arterial-embolisation Prognosis (HAP) score being calculated. Survival from diagnosis was calculated as well as the number of TACE procedures.

Results 268 patients were identified as having HCC treated with TACE in this period. $84.7 \%$ were male and $82.8 \%$ were cirrhotic. Patients all had BCLC stage A or B disease and most (94.1\%) Child Pugh A disease. Common causes of liver disease were: Alcohol (34.2\%), NASH (28.8\%) and Hepatitis C (10\%). HAP score was: HAP A (38.2\%), B (40.1\%), C $(19.5 \%)$ and $\mathrm{D}$ (2.2\%). Overall median survival for all patients receiving TACE was 862 days (IQR: 766-957). There was a significant difference in survival based on response after initial TACE (CR: 1284, PR: 840, SD: 820 and PD 306 days, $\log$ rank $\mathrm{p}<0.0001)$ and response after final TACE (CR: 1316, PR: 926; SD 1159 and PD 684 days, log rank $\mathrm{p}<0.0001)$. There was no difference in survival based on Child Pugh stage $(p=0.700)$ or if patients had BCLC A or B disease $(p=0.533)$.

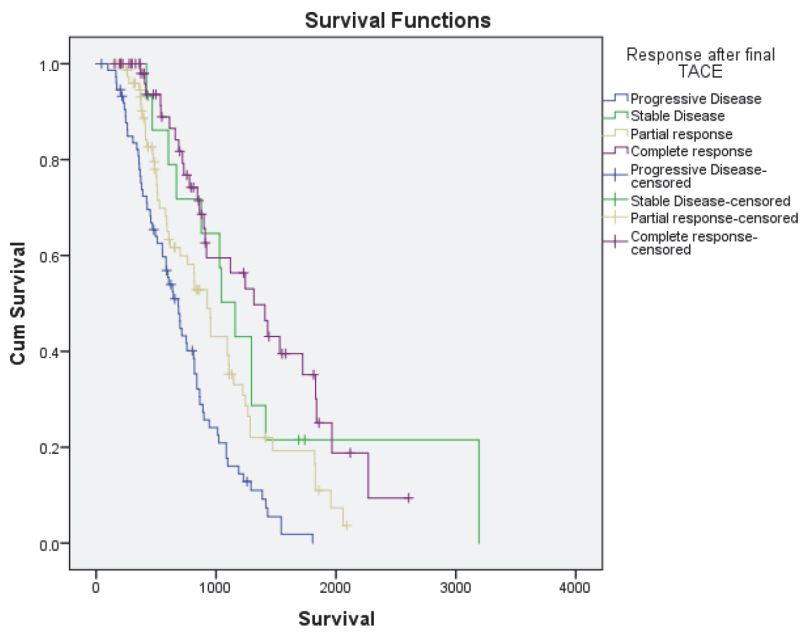

Abstract P185 Figure 1

Conclusions Our data suggests that patients' outcome following TACE for HCC relates to radiological response to treatment; those with CR having the longest survival and those with PD the shortest. Interestingly, those with PR and SD have similar outcomes based on response at first TACE and final TACE. Those with earlier stage disease (BCLC A) have similar outcomes to more advanced disease (BCLC B) suggesting that treatment determines outcome rather than initial stage of disease.

\section{P186 LONG TERM ABDOMINAL DRAIN FOR REFRACTORY ASCITES: ROYAL DERBY HOSPITAL EXPERIENCE}

Mohamed Elnagar*, Adam Lawson, Nicholas Taylor, Andrew Austin. Royal Derby Hospital, Derby, UK

\subsection{6/gutjnl-2020-bsgcampus.261}

Introduction In those patients who are not a candidate for liver transplantation or TIPSS, the palliation of refractory ascites is challenging. Repeated large volume paracentesis (LVP) is effective but requires hospitalization. Long term abdominal drains (LTAD) have been considered as an alternative to minimize the need for admission and improve quality of life. 